\title{
Whole genome sequencing in the investigation of recurrent invasive Group A streptococcus outbreaks in a maternity unit
}

Running title: Recurrent iGAS outbreaks in a maternity unit

Harriet Dickinson ${ }^{1}$, Mark Reacher ${ }^{1}$, Bernadette Nazareth ${ }^{2}$, Heidi Eagle ${ }^{3}$, Deirdre Fowler ${ }^{3}$, Anthony Underwood ${ }^{4}$, Meera Chand ${ }^{4,5,6}$, Victoria Chalker ${ }^{4}$, Juliana Coelho ${ }^{4}$, Roger Daniel ${ }^{4}$, Georgia Kapatai ${ }^{1}$, Ali Al-Shabib ${ }^{4}$, Richard Puleston ${ }^{1,7}$

Eastern Field Epidemiology Unit, National Infection Service, Cambridge ${ }^{1}$, Anglia Health Protection Team ${ }^{2}$, North West Anglia NHS Foundation Trust, Executive and Infection control teams ${ }^{3}$, PHE Microbiology Reference Services, National Infection Service ${ }^{4}$, Guy's \& St Thomas Hospitals NHS Foundation Trust ${ }^{5}$, NIHR Health Protection Research Unit in Respiratory Infections at Imperial college London ${ }^{6}$, University of Nottingham, School of Medicine, Division of Epidemiology and Public Health ${ }^{7}$.

Meera Chand and Victoria Chalker are affiliated with the National Institute for Health Research Health Protection Research Unit (NIHR HPRU) in Respiratory Infections at Imperial College London in partnership with Public Health England (PHE). The views expressed are those of the author(s) and not necessarily those of the NHS, the NIHR, the Department of Health or Public Health England.

Corresponding author: Richard Puleston

Public Health England

Seaton House

City Link

Nottingham

NG24LA

Tel:0344-2254524

richard.puleston@phe.gov.uk

\section{Summary}

\section{Background}

The clinical manifestations of Group A streptococcus (GAS) - (Streptococcus pyogenes) are diverse, ranging from asymptomatic colonisation to devastating 
invasive disease. Maternity related clusters of invasive Group A streptococcus (iGAS) infection are complex to investigate and control, especially if recurrent.

\begin{abstract}
Aim
We report on the investigation into three episodes of emm 75 GAS/iGAS infection in maternity patients at one hospital site over a 4 year period, two with monophyletic ancestry.
\end{abstract}

\title{
Methods
}

The episodes are described, together with whole genome sequence isolate analyses. Single nucleotide polymorphism differences were compared with contemporaneous emm 75 genomes.

\section{Findings}

Seven mothers had GAS/iGAS in over a 4 year period, emm 75, S.pyogenes and one had iGAS (in year 4) emm 3, S.pyogenes (subsequently discounted as linked). Three (clinical/screening samples) of the seven babies of emm 75 positive mothers and 3 screened healthcare workers were positive for GAS emm 75. Whole genome sequence similarity suggests a shared ancestral lineage and suggested a common source transmission but directionality of transmission cannot be inferred. However the findings indicate that persistence of a particular clone in a given setting may be long-term.

\section{Conclusions}

Occupational health procedures were enhanced, staff were screened and antibiotic therapy provided to GAS positive staff and patients. The definitive source of infection could not be identified, although staff / patient transmission is the most likely route. The pattern of clonal GAS transmission over 4 years suggests long-term persistence of GAS may have occurred.

Keywords. iGAS, Streptococcus, maternity, whole genome sequencing

\section{Introduction}

Group A Streptococcal infections have a spectrum of clinical manifestations (asymptomatic colonisation to severe invasive infections) occurring sporadically or in clusters. The incidence of invasive infection ranges from 1-3 cases/100,000 population, with a case fatality of $\sim 17 \%[1,2]$. Transmission is mostly via respiratory droplets/discharges or infected skin lesions ${ }^{[3]}$. Most GAS isolates are from the throat, but skin, vaginal, anal and perineal carriage have been noted; asymptomatic throat carriage is $5-12 \%{ }^{[4-6]}$.

Onward transmission can be reduced by robust infection prevention and control, prompt treatment of infection in cases and decolonisation through chemoprophylaxis in prolonged close contacts and colonised mother / neonate contacts. Wider chemoprophylaxis may be appropriate in some outbreaks, e.g. closed communities. 
After a hospital acquired iGAS case, the probability of a second in the same hospital is $\sim 40 \%$ within 12 months and $9.3 \%$ of hospital associated GAS infections are linked to others ${ }^{[1]}$.

There is a particular association between iGAS and the peripartum / postpartum period $[1,7]$. The age-specific attack rate for peripartum / puerperal period GAS is 20 times that of non-pregnant females ${ }^{[8]}$. In 2003-2004, 2-11\% of all severe GAS infections were associated with childbirth. Likewise between 1997-1999, puerperal GAS constituted $2 \%$ of all invasive GAS infections in the US/Canada. $[5,7]$. Pregnancy associated iGAS occurs mostly in the peripartum, but has been reported during pregnancy and the later postpartum period. Peripartum iGAS carries a substantial maternal morbidity / mortality burden (25\%), however the risk to the baby is greatest in antenatal iGAS, but still poses a substantial hazard to the new-born ${ }^{[9]}$.

GAS strains can be distinguished by emm gene sequence analysis. In England between September 2015-March 2016, the most common emm types associated with iGAS were emm $1(30 \%), 12(12 \%)$ and $89(11 \%)^{[10]}$.

Puerperal sepsis is fortunately rare. Indeed there are few reports of outbreaks in maternity settings published in recent years, however they may remain unrecognised; outbreaks have been associated with infected healthcare workers (staff-members) and shared bathroom facilities ${ }^{[11-13]}$.

Two clusters and an isolated case of infection with emm 75 GAS were noted over a 4 year period in an acute hospital maternity unit providing in/outpatient and community maternity services. (These are hereafter named as the initial cluster, the isolated case 2 years later and last cluster 4 years after the initial cluster). We report the investigations into the source, the control measures instigated and the application of whole genome sequencing (WGS).

\section{Methods}

Outbreak control teams for each episode were established to investigate the potential source and implement control measures.

\section{Case definitions}

\section{Confirmed}

A maternity staff-member/associated HCW working at the unit, or a maternity inpatient or baby born at the hospital in the 4 year period when cases occurred, with a confirmed isolate of emm 75 GAS from a clinical, i.e. infected or screening isolate, or colonised, isolate.

\section{Probable}

A maternity staff-member/associated HCW working at the unit, or a maternity inpatient or baby born at the hospital in the 4 year period when cases occurred, who had a 
suspected GAS infection and a GAS isolate identified or colonised with a GAS isolate, but with a non-emm 75 strain or no typing data available.

The initial probable case definition was kept broad to include any emm typing positive isolate to maximise case capture until the situation was clear. However, any non-emm 75 clone isolates/cases were subsequently discounted as being part of the outbreak.

\section{Possible}

A HCW from/inpatient admitted to, another part of the hospital, with suspected or confirmed GAS infection of any type/strain in the hospital in the 4 year period when cases occurred, with suspected GAS infection but without microbiological confirmation or typing data/matching emm type.

\section{Incidents}

Over the 4 year period, 7 mothers, 3 neonates and 3 Staff-members who were all confirmed, clinical cases, were reported with GAS/iGAS emm 75 infection. One other mother from the last cluster had emm 3 and was subsequently discounted as being part of the outbreak. Six mothers had iGAS and 1 (the index) had GAS infection, One baby and 3 Staff-members had non-invasive disease and 2 babies were colonised only. There were no deaths. The mothers (aged 22-39 years) received all their maternity care from the hospital; their babies were 1-44 days old at diagnosis. No neonatal cases were delivered by caesarean section. All mothers were well at admission for delivery, although one had an elevated white blood cell count. Three had recognised risk factors, other than pregnancy.

In the initial cluster, the index case re-presented to hospital with postnatal complications; GAS was identified from vaginal sampling. Over the next 5 days, 3 maternal iGAS cases occurred. One baby of these mothers developed a GAS skin infection, within 10 days of the presentation of the index, another was found to be colonised and the baby of index was found to have a positive throat swab 7 days after the presentation of the index. All cases in this initial cluster had emm 75 isolates. A patient with puerperal sepsis also presented 10 days after the index, but no microbiological isolates were available for that case.

A single, maternal, confirmed case of emm 75 iGAS was identified 2 years after the initial cluster, followed by 2 confirmed maternal emm 75 iGAS cases 4 years later in the final cluster (symptomatic within 6 days of each other) and a single maternal emm 3 case, who became symptomatic 20 days after the first case in this last cluster. This latter case was subsequently discounted as being part of the outbreak. There were no neonatal cases in associated with the isolated case that occurred 2 years after the initial cluster or in the last cluster.

\section{Investigations and control measures undertaken}

Cases and their close contacts were treated as per standard guidelines. 
Staff screening sampling results were obtained. GAS positive staff were excluded from work, treated and rescreened for clearance. Wider staff prophylaxis was not employed.

For the last cluster, risk factors and symptoms, microbiology results and patient-staff interactions (dates/locations) were obtained. Ward movements were examined for staff/case overlaps and mapped against staff sickness absence records. Genome sequencing and emm sequence typing were undertaken. Environmental sampling was undertaken on the delivery suite after a deep clean had occurred and a month later (specific sampling site details are not available).

\section{Microbiological investigation}

\section{Emm gene typing method}

The emm types were determined using the Centers for Disease Control protocol (table 1). When sequence data obtained were ambiguous, alternative primers (MF1, $5^{\prime}$ ATAAGGAGCATAAAAATGGCT-3', and MR1, 5'-AGCTTAGTTTTCTTCTTTGCG-3') were used ${ }^{[14]}$.

Genome sequencing and single nucleotide polymorphisms (SNP) calling was performed on 13 GAS isolates from the hospital and contemporaneous emm 75 isolates from England as previously described using the emm 2 MGAS10270 reference genome, RefSeq accession number NC_008022.1 as the closest available GenBank sequence ${ }^{[15]}$. This was used in the absence of an emm 75 reference genome using bwa-mem 0.7.5a ${ }^{[16]}$. Gubbins 1.3.3 was used to remove loci with elevated base substitution densities indicative of recombination, producing a multiple sequence alignment file containing only non-recombining variant positions [17]. These data were used to generate a maximum-likelihood tree using RAxML version 8.1.17 using the following parameters:

-f a (rapid Bootstrap-analysis and search for best-scoring ML tree in one program run) -m (substitution-model) GTRGAMMA

-p (random-seed for parsimony inferences) 12345

-x (random-seed for bootstrapping) 12345

\# (number of runs) $1000{ }^{[18] .}$

\section{Results}

Staffing sickness absence was available for the last cluster only however staff sampling results from the initial cluster (nose/throat swabs only), isolated case 2 years later (sample sites unknown) and last cluster (nose/throat,$+/$ - perineum sampling if first nose/throat screen was positive) incidents were obtained.

In the initial cluster, at least 170 staff were screened with throat swabs/cultured for GAS; the specialities of the staff sampled were not known; one was positive for GAS emm 75 and had become symptomatic with throat symptoms only after all cases were diagnosed excepting the baby of the index, who was detected on screening. 
With the isolated case 2 years later, 54 staff were documented as appropriate to screen by throat/wound swabbing and culture for GAS. These included 32 maternitystaff with the remainder from other teams e.g. ITU. Of the 54 eligible staff, 35 were sampled with 25 being from the maternity department and all were screen negative.

In the final cluster, staff screening was undertaken over 3 months. Throat/wound swabs were taken initially with the subsequent addition of nose and perineum samples for those screened later. In the first round, 371 staff (208 maternity-staff) were sampled, and in the $2^{\text {nd }}$ to $4^{\text {th }}$ rounds, 85,78 and 72 (37, 35 and 31 maternity staff) were repeat sampled respectively; the number eligible for sampling was not known. Although 4 staff-members were GAS positive on screening, only one maternity staffmember had the emm 75 strain and became unwell 4 days after the second emm 75 case in the last cluster, but before the emm 3 case. The others had strains unrelated to the outbreak. Additionally, a symptomatic staff-member from a different department was identified as having an emm 75 isolate 3 months later. This suggests emm 75 may have been circulating more widely in other staff within the hospital and supports the hypothesis that staff were the unwitting source in these incidents.

\section{Routes of transmission}

Four possible transmission routes were investigated:

\section{Mother-to-mother transmission}

No mother was present in more than one cluster (i.e. did not deliver a baby at the maternity unit in each of the affected year clusters); it is therefore unlikely that an individual chronic carrier mother could have been the primary vector responsible for mother-to-mother transmission in all three clusters. Two overlapped for one day in the initial cluster on the delivery suite but were in separate rooms; all 4 had been in the maternity unit within a 7 day period. The first and second mothers in the last cluster overlapped in the delivery-suite on the day the first was readmitted with a positive GAS isolate, occupied the same delivery room during delivery and same postnatal ward bay but with a 3 day separation respectively.

\section{Staff-patient-staff transmission}

Emm 75 was found on staff-screening in the initial cluster, with one maternity staffmember found to be colonised and in the last cluster, again with one maternity staffmember found to be infected plus an additional non-maternity unit staff-member.

No direct link between a single staff-member and all cases was identified; however two staff had contact with one or more cases in each of the episodes.

The staff-member who was GAS positive in the initial cluster was present and cared for 5 of the cases then, but only became symptomatic after all the cases had presented, except the baby of the index whose isolate was detected on screening. This staff member was also involved in the care of the single case 2 years later, but was not present for the last cluster. The GAS-positive maternity staff-member in the last cluster had contact with the index case of that last cluster $>2$ weeks before onset, but didn't develop symptoms until after the last cluster index case had presented, but did have contact with 3 of the cases ( 2 mothers, 1 baby) in the initial cluster within 3 
days of their infection being identified. One staff-member was common to 2 cases in the last cluster and another to all 3 cases in that cluster, but no positive isolates were obtained from them.

\section{Staff-staff transmission}

In the last cluster, some staff-members had worked while symptomatic with potential GAS infections, e.g. sore-throat. However, this was only after the index and second case in the last cluster had already presented, making it less likely they were the primary vectors (more likely to have been downstream recipients). In the first round of screening in the last cluster, 371 staff were sampled; follow-up sampling was attempted for a further three-rounds to try to increase detections, however fewer were re-sampled on each occasion, 85, 78 and 72 respectively, meaning that other asymptomatic staff who were not sampled could have been the primary vectors, but remained undetected, or that where negative results were obtained that the organisms present were below the limit of detection.

\section{Environmental transmission}

During the investigations of the last cluster the unit was examined for infection prevention and control compliance; some deficiencies were noted, e.g. blood contaminating a privacy curtain, clinical equipment placed on waste bin tops. Recommendations for improvements were made. GAS was not isolated from environmental sampling (however the sites sampled were not available to the outbreak team, so the appropriateness of sampled sites or the sampling methods used was not known).

\section{Genomic sequencing analysis}

Whole genomic sequence analysis showed the initial and last cluster isolates were located within a clade with a shared ancestral lineage suggesting common source transmission; however transmission directionality could not be inferred. The isolates were separated from all others by at least 9 SNPs. No other contemporaneous isolates from national emm 75 GAS strains were located within this clade (Figure 1). The single case isolate that arose 2 years after the initial cluster did not have an immediate common ancestor to the initial or last cluster isolates.

\section{Discussion}

No mother was present in more than one cluster; it is therefore unlikely that an individual chronic carrier mother could have been the primary vector responsible for mother-to-mother transmission in all the clusters. However mother-to-mother transmission may have occurred within the individual cluster, e.g. 2 mothers overlapped in the initial cluster on delivery suite for one day but had no direct contact, making this less likely and in the last cluster two occupied the same bay/bathroom and delivery-suite room but on different days. It is also unlikely the mothers were separately importing GAS leading to multiple re-introductions into the hospital environment as there was little evidence of circulating community emm 75 ; nationally only $3.2 \%$ and regionally $3 \%$ were emm 75 during the period and all of the regional emm 75 isolates 
resulted from this incident, suggesting emm 75 is relatively uncommon in invasive disease.

The recurrence of clonal GAS clusters over such an extended 4 year period is unusual. The initial and final cluster isolates were closely related, with a common ancestor and are distinct from other emm 75 isolates. The variation observed between the initial and final cluster isolates is indicative of acquired variation consistent with genetic drift over time. The number of single point mutations (single nucleotide polymorphisms or SNPs) estimated to occur in the GAS genome (based on analysis of emm 1/12 type strains) has been estimated at 1.37 to 1.8 SNPs per core genome per year. ${ }^{19,20]}$. The genetic distance between the initial and last cluster maternity unit clade of isolates is consistent with these estimates. The 9 SNPs that separate the maternity unit clade from all other isolates in this study represent an approximate time of 6.5 years.

It is possible the initial cluster strain could have persisted in the hospital environment and/or in one or more colonised staff until the last cluster; however such a longduration (greater than two years) of clonal-persistence has not been previously described.

There are three possible explanations for the emergence of a second clonally linked cluster four years after the first infection episode:

1. Treatment / screening failure in the initial cluster with one or more staffmembers retaining asymptomatic emm 75 GAS colonisation/shedding, leading to ongoing staff-staff, staff-patient, staff-environment transmission. It has previously been demonstrated that asymptomatic carriage is less effectively cleared by treatment, than for symptomatic cases ${ }^{[21]}$.

2. A persistent external source/s reintroducing GAS to the hospital, e.g. staffmembers / relatives.

3. Persistence of environmental contamination. GAS has been documented to persist in fomites for up to $61 / 2$ months ${ }^{[22]}$.

Definitively confirming any of these hypotheses is not possible, however the first is the most plausible, since staff colonisation was found, no inanimate reservoir was detected and emm 75 GAS / iGAS disease is rare. Supporting this; asymptomatic, colonised staff-members and symptomatic staff-members have been previously demonstrated to account for $34 \%$ and $8.2 \%$ of all GAS outbreaks respectively and carriage with the same strain in close contacts of a GAS-infected case can approach $30 \%{ }^{[5,23]}$. Staff may have unwittingly transmitted GAS to each other leading to persistence in multiple staff-members. Control measures were therefore targeted at interrupting transmission from staff, including the screening of staff to detect carriers, attention to handwashing and infection control procedures.

WGS was useful for supporting the linkage of cases and determining that the clusters were genetically distinct from other emm 75 isolates, however more contemporaneous isolates would have been helpful to indicate more clearly the incidence of infections / prevalence of carriage of this particular clade. 
Although staff screening was undertaken, the logistics of sampling and particularly repeat sampling a large group of individuals was challenging; screening effectiveness is also limited by sample quality and false negative results. Data quality was also an issue; data on the location of mothers was incomplete, not all staff-patient interactions were documented and staff signatures were frequently unclear. Increased duration and proximity of staff / patient interactions may increase the chances of transmission; however it is unusual for these details to be noted in clinical records.

While environmental surfaces have been recognised as the source of maternity GAS outbreaks, for example, GAS remain viable on fomites for up to $6 \frac{1}{2}$ months and shower heads, curtains, baths, bidets and toilet seats have been previously implicated and transmission vectors, and there were some infection control deficiencies identified, sampling nonetheless found no evidence of environmental GAS contamination [5, 22, 24]. Environmental sampling was performed with dry swabs after deep-cleaning which may have reduced detections. The sites sampled were also not available to the outbreak team so their relevance was not known. Moistened / specific environmental swabs or directly applied plates may have produced more meaningful results ${ }^{[24]}$. Although speculative in the absence of clearer environmental sampling data, it is unlikely a single persistent environmental source was responsible due to the time between the two episodes. It is more likely the environment acted as a temporary, intermediate vector. Even with systematic, environmental sampling using moistened swabs, areas of contamination may be missed, so negative swabs are not necessarily indicative of a non-contaminated environment. The focus therefore should be on assuming that there may be temporary contamination of the care environment and robust cleaning to reduce such contamination to a minimum. GAS prevention and control guidelines recommend 'communal facilities, including bathrooms / toilets, are cleaned and decontaminated after each use especially on delivery-suites, post-natal wards and other high-risk areas' ${ }^{[5]}$. At the time of the last cluster the cleaning regime was consistent with other NHS practice, namely twice daily cleaning, however cleaning was subsequently increased to decontaminate after each patient use. Further research on the optimal cleaning frequency for such high risk areas is warranted.

\section{Conclusions}

Recurrent clonal outbreaks of GAS/iGAS spanning comparable durations to this incident and emm 75 outbreaks related to childbirth appear rare. We postulate that recurrent infections seen with isolates within the emm 75 monophyletic group occurred over a four year period due to asymptomatic carriage in maternity staff-members, with transmission to patients. However, other modes of transmission cannot be excluded. Increased monitoring and vigilance is essential, particularly if more cases with the emm 75 type are identified. Staff and patient education will be a valuable tool in preventing any future cases. No further cases have been noted at the time of writing, (March 2018).

Authorities investigating maternity related outbreaks should be aware that prolonged, clonal isolate outbreaks are possible over a period of years, indicating that robust efforts to identify, control and eradicate the common source are needed, particularly given the seriousness of puerperal sepsis. Fortunately all invasive cases recovered in these incidents, but some were very ill, so the outcome could have been very different.

\section{Acknowledgements}


We would like to thank Chenchal Dhami, Tim Chambers and Marisa Laranjeira for laboratory assistance, the hospital's infection prevention and control team, the outbreak control team and hospital staff.

\section{References}

[1] Daneman N, McGeer A, Low DE, Tyrrell G, Simor AE, McArthur M et al. Hospital-acquired invasive group a streptococcal infections in ontario, canada, 1992-2000. Clinical Infectious Diseases 2005; 41: 334-42.

[2] O'Loughlin RE, Roberson A, Cieslak PR, Lynfield R, Gershman K, Craig A et al. The epidemiology of invasive group a streptococcal infection and potential vaccine implications: United states, 2000-2004. . Clinical Infectious Diseases: An Official Publication of the Infectious Diseases Society of America, 45(7), 853-62 doi:101086/521264 2007; 45: 853-62.

[3] Health Protection Agency. Interim uk guidelines for management of close community contacts of invasive group a streptococcal disease. Streptococcus working group. 2004; 354-61.

[4] Shaikh N, Leonard E, Martin J Prevalence of streptococcal pharyngitis and streptococcal carriage in children: A meta-analysis. Pediatrics 2010; 126: e557e64.

[5] Steer JA, Lamagni T, Healy B, Morgan M, Dryden M, Rao B et al. Guidelines for prevention and control of group a streptococcal infection in acute healthcare and maternity settings in the uk. Journal of Infection 2012; 64: 1-18.

[6] Stamm WE, Feeley JC, Facklam RR Wound infections due to group a streptococcus traced to a vaginal carrier. Journal of Infectious Diseases 1978; 138: 287-92.

[7] Factor SH, Levine, O. S., Schwartz, B., Harrison, L. H., Farley, M. M., Mcgeer, A., \& Schuchat, A. Invasive group a streptococcal disease : Risk factors for adults. . Emerg Infect Dis 2003; 9: 970-7.

[8] Deutscher M, Lewis M, Zell ER, Taylor TH, Van Beneden C, Schrag S Incidence and severity of invasive streptococcus pneumoniae, group a streptococcus, and group b streptococcus infections among pregnant and postpartum women. Clinical Infectious Diseases 2011; 53: 114-23.

[9] Hamilton SM, Stevens DL, Bryant AE Pregnancy-related group a streptococcal infections: Temporal relationships between bacterial acquisition, infection onset, clinical findings, and outcome. Clinical Infectious Diseases: An Official Publication of the Infectious Diseases Society of America 2013; 57: 870-6.

[10] Public Health England Group a streptococcal infections: Fourth update on seasonal activity, 2015/16. Health Protection Report, weekly report 2016; 10.

[11] Ejlertsen T, Prag J, Holmskov A, Pettersson E A 7-month outbreak of relapsing postpartum group a streptococcal infections linked to a nurse with atopic dermatitis. Scandinavian Journal of Infectious Diseases 2001; 33: 734-7. 
[12] Gordon G, Dale BAS, Lochhead D An outbreak of group a haemolytic streptococcal puerperal sepsis spread by the communal use of bidets. BJOG: An International Journal of Obstetrics \& Gynaecology 1994; 101: 447-8.

[13] Claesson BEB, Claesson ULE An outbreak of endometritis in a maternity unit caused by spread of group a streptococci from a showerhead. Journal of Hospital Infection 1985; 6: 304-11.

[14] Podbielski A, Melzer B, Lütticken R Application of the polymerase chain reaction to study the $m$ protein(-like) gene family in beta-hemolytic streptococci. Medical Microbiology and Immunology 1991; 180: 213-27.

[15] Chalker V, Jironkin A, Coelho J, Al-Shahib A, Platt S, Kapatai G et al. Scarlet fever incident management team. Genome analysis following a national increase in scarlet fever in england 2014. BMC Genomics 2017; 18: 224.

[16] Bolger AM, Lohse M, Usadel B Trimmomatic: A flexible trimmer for illumina sequence data. Bioinformatics 2014; 30: 2114-20.

[17] Croucher NJ, Page AJ, Connor TR, Delaney AJ, Keane JA, Bentley SD et al. Rapid phylogenetic analysis of large samples of recombinant bacterial whole genome sequences using gubbins. Nucleic Acids Research 2015; 43: e15.

[18] Stamatakis A Raxml-vi-hpc: Maximum likelihood-based phylogenetic analyses with thousands of taxa and mixed models. Bioinformatics 2006; 22: 2688-90.

[19] Davies MR, Holden MT, Coupland P, Chen JHK, Venturini C, Barnett TC et al. Emergence of scarlet fever streptococcus pyogenes emm12 clones in hong kong is associated with toxin acquisition and multidrug resistance. Nat Genet 2015; 47: 84-7.

[20] Nasser W, Beres SB, Olsen RJ, Dean MA, Rice KA, Long SW et al. Evolutionary pathway to increased virulence and epidemic group a streptococcus disease derived from 3,615 genome sequences. Proceedings of the National Academy of Sciences 2014; 111: E1768-E76.

[21] Kaplan E, Gastanaduy A, Huwe B The role of the carrier in treatment failures after antibiotic for group a streptococci in the upper respiratory tract. $J$ Lab Clin Med 1981; 98: 326-35.

[22] Kramer A, Schwebke I, Kampf G How long do nosocomial pathogens persist on inanimate surfaces? A systematic review. BMC Infectious Diseases 2006; 6: 130 -.

[23] Weiss K, Laverdière M, Lovgren M, Delorme J, Poirier L, Béliveau C Group a streptococcus carriage among close contacts of patients with invasive infections. American Journal of Epidemiology 1999; 149: 863-8.

[24] Mahida N, Beal A, Trigg D, Vaughan N, Boswell T Outbreak of invasive group a streptococcus infection: Contaminated patient curtains and cross-infection on an ear, nose and throat ward. Journal of Hospital Infection 2014; 87: 141-4.

\section{Declarations}

Funding or sponsorship: This work was funded by Public Health England.

Conflicts of interest: HD is currently employed by GlaxoSmithKline and holds GSK shares. At the time this work was done she was employed by Public Health England. 